\title{
COUPLING OPTIMIZATION OF POINT CONTACT DETECTORS AND MIXERS
}

J.Ch. BOLOMEY, J. CAHSMAN, S. EL HABIBY (1)

M. PYEE (2)

A. COMERON, J.C. HENAUX, J.J. JIMENEZ, R. ADDE, G. VERNET (3)

(1) Laboratoire des Signaux et Systèmes - Groupe d'Electromagnétisme (CNRS-ESE) Plateau du Moulon, 91190 GIF-sur-YVETTE - FRANCE

(2) Labo. de Résonance Magnétique et d'Electronique Quantique. Univ. P. et M. Curie, 4, place Jussieu, 75230 PARIS CEDEX 05 - FRANCE

(3) Institut d'Electronique Fondamentale. Univ. de Paris-XI, Centre d'Orsay, Bât.220, 91405 ORSAY FRANCE

GRECO MICROONDES

One shows that, for a given IR power, the angular width of an illuminating focused laser beam can be adjusted to optimize the electromagnetic coupling. The predicted conditions have been checked experimenta1ly for various devices (Schottky, MIM, Josephson) at 337,70 and $10.6 \mu \mathrm{m}$.

In the IR range, point contact structures provide good performances until wavelengths of few microns.The coupling to external fields occurs in the free-space, the point contact structure being used alone or in conjunction with mirrors of various shapes (corners, parabolic cylinders...). The external fields consist of focused or parallel laser beams.

The coupling problem is to deliver to the junction located at the tip of the point contact struc ture the largest possible part of the power carried by the incident beams. For a few years ago, this problem has been considered as an antenna problem [1] [2] [3]. This point of view has allowed to determine the incidence angle providing an efficient coupling. A more complete electromagnetic treatment [4] [5] includes more details on the illumination conditions and it is then possible to predict quantitatively the effect of various parameters of practical interest such as focusing angle, defocusing..

The consideration of the point contact structure as an antenna leads us to characterize it by its radiation pattern $\vec{F}(\vec{u})$ in the direction $\vec{u}$ and its input impedance $Z_{i}$ in the emitting situation. Characterizing a laser beam by its angular spectrum of spherical waves has proved to be very convenient, especially for focused beams [5]. The particular case of a parallel beam can also be considered.The angular spectrum $\vec{E}^{+}(\vec{u})$ associated to the incoming spherical wave of the beam is a quantity describing the angular variations of the field distribution in the cross section of the laser beam. Similarly $\vec{F}(\vec{u})$ describes the angular variations of the far field which would be radiated by the structure in emitting situation.

It has been shown that the power coupling factor $\rho$, defined as the ratio of the power delivered to the junction-characterized by its equivalent impedance $Z_{j}$ - to the incident power, is given by [4]:

$$
\begin{aligned}
& \rho_{L}=\rho_{L} \rho_{E} \\
& \rho_{L}=1-\left|\frac{z_{j}-z_{i}^{*}}{z_{j}+z_{i}}\right|^{2} \\
& \rho_{E}=\frac{\left|\int_{4 \pi} \vec{E}^{+}(\vec{u}) \cdot \vec{F}(\vec{u}) d \omega(\vec{u})\right|^{2}}{\int_{4 \pi} E^{1+2}(\vec{u}) d \omega(\vec{u}) \cdot \int_{4 \pi} F^{\prime 2}(\vec{u}) d \omega(\vec{u})}
\end{aligned}
$$

where $d_{\omega}(\vec{u})$ denotes the elementary solid angle in the direction $\overrightarrow{\mathrm{u}}$, and $\mathrm{A}^{\prime 2}$ is the local hermitian norm of a vector A.

The load factor $\rho_{\mathrm{L}}$ and the electromagnetic factor $\rho_{E}$ vary between $O$ and 1 . The value 1 is reached under the following conditions :

$$
\begin{aligned}
& z_{j}=z_{i}{ }^{*} \\
& \vec{E}^{+}(\vec{u})=\vec{F}^{*}(\vec{u}) \quad ; \forall \vec{u}
\end{aligned}
$$

The first condition is well known from classical circuit theory, the second one indicates that the angular distributions of $\vec{E}^{+}$and $\vec{F}$ are identical and that the corresponding vectors describe conjugate ellipses (polarization match). When both conditions (4) and (5) are verified, the power transfer from the beam to the junction is total. In practice, it is difficult to satisfy exactly these conditions, but it is always to use (1-3) to compute $\rho$ for any given configuration. This has been done for Josephson junctions at $337 \mu \mathrm{m}$ and predicted values are in close agreement with experimental results.

Relating $\vec{F}$ and $\vec{E}+$ to the practical parameters of the structure allows to point out their influence on $\rho_{E}$. An idealized situation is illustrated in Fig. 1. Assuming that $\vec{F}$ and $\vec{E}^{+}$can be approximated by pulse-type functions (constant in a given angular interval and zero outside) and there is a perfect polarization match, one obtains when $\theta_{i}=\theta_{s}$.

With

$$
P_{E}=\frac{\Delta \phi_{i}}{2 \pi} \frac{\widetilde{\Delta \Theta}^{2}}{\Delta \Theta_{i} \Delta \Theta_{s}}
$$

$$
\tilde{\Delta \oplus}=\operatorname{Min}\left\{\Delta \Theta_{i}, \Delta \Theta_{S}\right\}
$$

Where $\Delta \theta_{i}, \Delta \phi_{i}, \Delta \theta_{S}, \theta_{i}$ and $\theta_{S}$ are indicated in Fig. 1. For instance, for long thin wire structures of length $l$ at wavelength $\lambda: \Delta \theta_{S} \simeq \sqrt{l}$

For laser beams focused by lenses of aperature $D$ and focal length $f: \Delta \theta_{i} \simeq \frac{\mathrm{D}}{2 \mathrm{f}}$

Clearly, concerning the azimuthal aspect, the factor $\rho_{E}$ is maximum for $\Delta \theta_{i}=\Delta \theta_{S} \quad$ (Fig. 2). This 
condition defines the optimal coupling.

$$
\rho_{E, \text { p t }}=\frac{\Delta \phi_{i}}{2 \pi}
$$

formula shows the interest of distroythe structure pating the rotationnal symmetry ing by means of mirrors $[6]$. In that case the facthe azimutal angular widt of the structure pattern.

The optimal illuminations cheked experimenty with have been successfully cheke $6 \mu \mathrm{m}, 70 \mu \mathrm{m}$ and 337 MIM and Schottky diodes at $10.6 \mu \mathrm{m}$, Im [7] [8]. Fig. 3 shows a typical variation of the coupling factor versus beam angular width obtained by ung lenses of different focal length. This re by using lenses of difo rad sult corresponds to a schottky diode

tion pattern at $337 \mu \mathrm{m}$ is given in

It can be easily taken into account the defo calization effects by means of the translation the rem. If $\vec{\delta}$ is the tratslation vector relaying the tip of the structure to the formula (3) for the tern $\vec{F}$ has to be changed in formula

translated pattern $\vec{F}_{\delta}$ such that :

In any case, expressions (1-3) appear to be very un order to calculate the power couin ortern of pling factor as long as the radiation patter known. the structure and its input impedance are known.

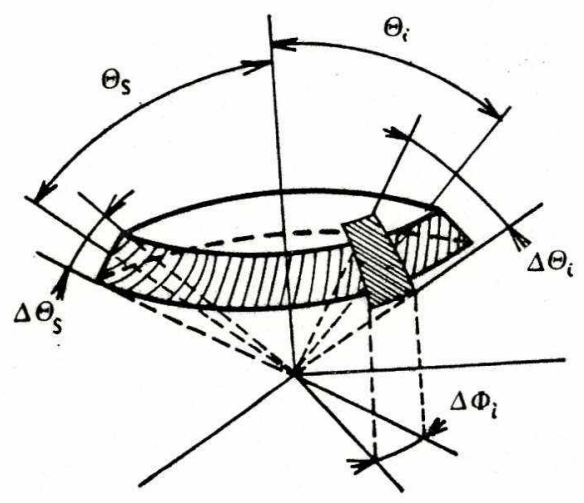

1 Dlland of a laser beam by its re by its pattern

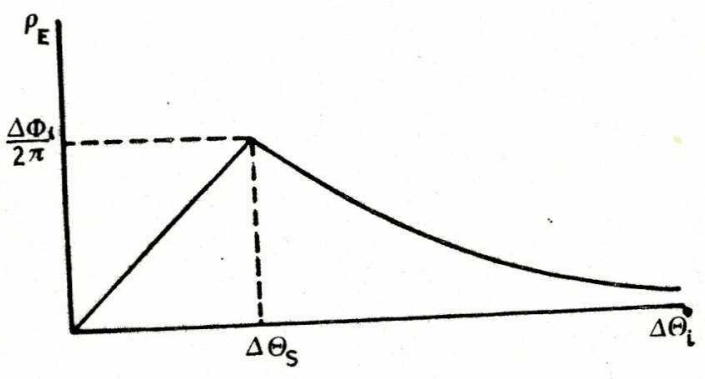

. Idealized variation of the coupling FIGURE 2 : Idealized variaticient versus angular beam width $-\left(\theta_{i}=\theta_{S}\right)$.

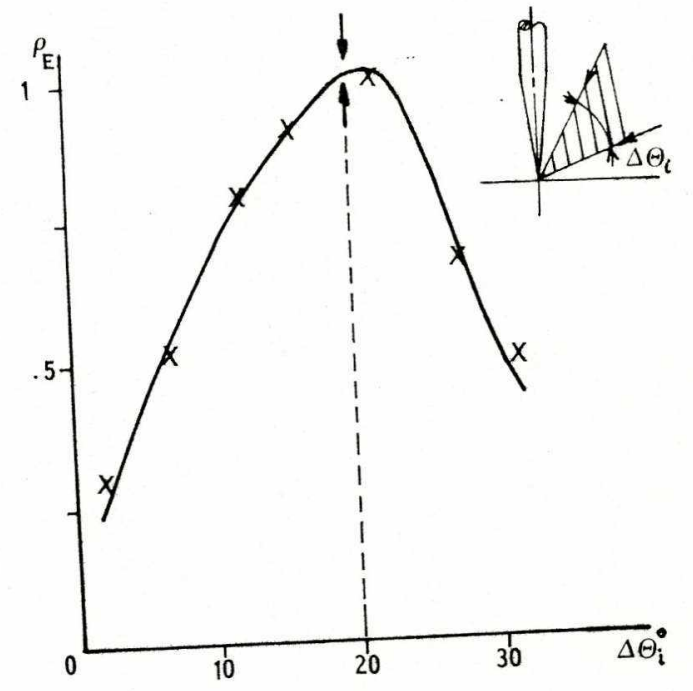

FIGURE 3 : Coupling factor variation (in arbitrary FIGURE $3:$ Coupling factor variation $-\theta_{j}=\theta_{S}$ ) versus beam angular width. The optimal coupling $\left(\Delta \theta_{i}=\Delta \theta_{S}\right)$.

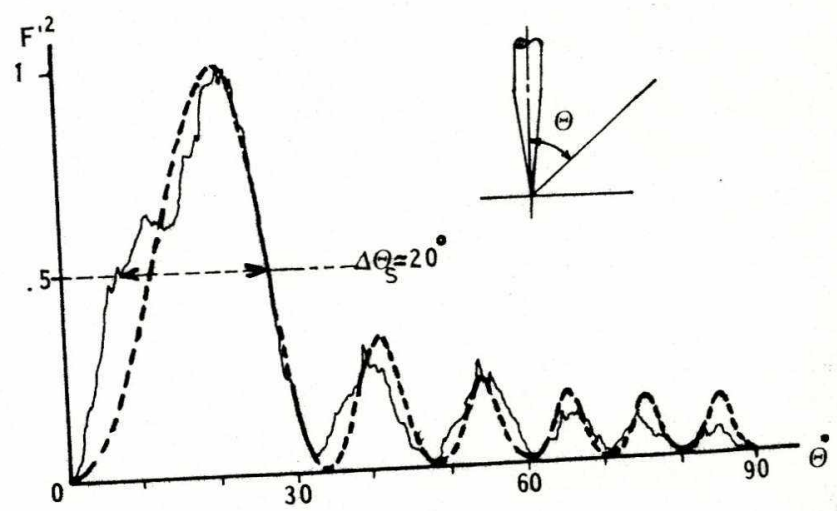

FIGURE 4 : Normalized radiation pattern of a Schottky diode at $337 \mu \mathrm{m}$.

experimental theoretical (using current approximation $[1]$ )

EIBL TOGRAPH Y

[1] MATARRESE L.M., EVENSON K.M., App1.Phys.Lett. Vo1.17, $\mathrm{n}^{\circ} 1,1$ ju1y 1970, pp $8-10$

SCHWARZ S.E., Appl. Phys. Lett., vol. $26, \mathrm{n}^{\circ} 12$, june $1975, \mathrm{pp} \cdot 672-675$.

[3] SULLIVAN T.E., CUTLER P.H., LUCAS A.A., SU5ce Science, vol. $62, \mathrm{n}^{\circ} 2, \mathrm{Feb} .77, \mathrm{pp} \cdot 455-$ 471 .

[4] BOLOMEY J.Ch., Int. Conf. Ant. and Prop., London 28-30 Nov. 1978 .

[5] BOLOMEY J.Ch., Annales des Tele To be published.

[6] KRAUTLE ' , SAUTER E., S, Nov. 1977

Physizs 101.17 , n

[7] BOLOMEY J Ch. et al. , Final Report ATP-D2937, May 77- May 79.

8] PYEE M., Proc. BRSG Symp.,Keele, April 1978. 